\title{
An Improved Grey Model with Time Power and Its Application
}

\author{
Jianming Jiang $\mathbb{D},{ }^{1}$ Caixia Liu $\mathbb{D},{ }^{2}$ Yuanguo Yao $\mathbb{D},{ }^{1}$ Yumu Lu $\mathbb{D},{ }^{1}$ Wanli Xie $\mathbb{D}^{2},{ }^{2}$ \\ and Chong Liu iD ${ }^{3}$ \\ ${ }^{1}$ School of Mathematics and Statistics, Baise University, Baise 533000, China \\ ${ }^{2}$ Institute of EduInfo Science and Engineering, Nanjing Normal University, Nanjing 210097, China \\ ${ }^{3}$ College of Sciences, Northeastern University, Shenyang 110819, China
}

Correspondence should be addressed to Yuanguo Yao; 44285843@qq.com

Received 22 April 2020; Revised 3 November 2020; Accepted 5 January 2022; Published 29 January 2022

Academic Editor: Qingdu Li

Copyright ( $\odot 2022$ Jianming Jiang et al. This is an open access article distributed under the Creative Commons Attribution License, which permits unrestricted use, distribution, and reproduction in any medium, provided the original work is properly cited.

The grey system model with time power, which is often called the $\operatorname{GM}\left(1,1, t^{\alpha}\right)$, appeals considerable interest of research due to its effectiveness in time series forecasting. Aimed to improve further the $\operatorname{GM}\left(1,1, t^{\alpha}\right)$ model, this paper introduces a new whitening equation with variable coefficient into the original whitening equation which extends applicable scope; as a result, an improved grey model with time power, namely, $\operatorname{OGM}\left(1,1, t^{\alpha}\right)$, is proposed. Firstly, the time response function of the novel model and the restored values of original series are deduced through grey modelling techniques. Secondly, the variable coefficient in the whitening equation and the time power are determined by particle swarm optimization algorithm. Two empirical examples are then used to verify the validity of the novel model. Finally, the novel model is applied to predict the oil consumption of China from 2004 to 2018. Results show the novel model outperforms other commonly-used competitive models, which can well serve a benchmark model for scholars and decision-makers.

\section{Introduction}

With the deep research of theoretical methods and the extension of application fields, the grey prediction model is gradually applied to predict the fluctuation characteristics from original tendency prediction [1]. Grey forecasting model, as an important branch of grey system theory [2], namely, $\operatorname{GM}(1,1)$, has been widely used in various fields, including energy [3], economic, and engineering. Among them, for example, Ding [4] investigated the natural gas consumption of China based on a novel approach of combining a new initial condition and rolling mechanism for enhancing the prediction accuracy. Wang et al. [5] explored the nonlinear relationship between carbon dioxide emissions and economic growth through constructing a PSO algorithm-based grey Verhulst model. Wu et al. [6] forecasted carbon dioxide emissions in the BRICS countries using a novel multivariate grey model. Wang et al. [1] proposed NLS-based nonlinear grey Bernoulli model to predict employee demand of high-tech enterprises in China.
Kumar et al. [7] utilized time series models, including greyMarkov, grey model with rolling mechanism, and singular spectrum analysis, to forecast energy consumption in India. Duamn et al. [8] predicted e-waste for reverse logistics operations based on improved univariate grey models. Ma et al. [9] developed a novel time-delayed polynomial grey model to predict the natural gas consumption in China.

Under this context, grey forecasting model has been generally received more attention in recent years, numerous grey extensive models have been deduced. For instance, Xie and Liu [10] put forward a discrete grey model which was abbreviated as $\operatorname{DGM}(1,1)$; in addition, he also depicted the connection between $\operatorname{DGM}(1,1)$ and $\operatorname{GM}(1,1)$, which extends applicable scope. Luo and Wei [11] proposed a grey model with polynomial term and its optimization methods. Afterward, Wei et al. [12] estimated the optimal solution for novel grey polynomial prediction model. Wang et al. $[13,14]$ proposed a series of methods of handling with seasonally fluctuating sequence with small-size characteristics. Wu and Zhang [15] proposed an improved time series interval forecasting method 
inspired by the literature [16]. Qian et al. [17] proposed a grey model with time term (short for $\mathrm{GM}\left(1,1, t^{\alpha}\right)$ ).

Considering the nonlinear characteristics hidden in original series, Chen et al. [18] firstly introduced the Bernoulli model into the traditional grey action quantity, so as to capture the nonlinear trend in raw sequences. Later, Wang et al. [19] optimized this model through changing the background-value coefficient. Wu et al. [20] simultaneously combined a new initial condition and dynamic background value in order to improve further $\operatorname{NGBM}(1,1)$ model and applied it to predict China's GDP. Nguyen et al. [21] proposed nonlinear grey Bernoulli model based on Fourier transformation. Ma et al. [22] propounded a novel nonlinear multivariate grey Bernoulli model to predict the tourist income of China.

As many studies reveal, it is a common sense that the fractional accumulation is an effective method to improve further the grey model. Wu et al. [23] originally proposed a grey model with fractional grey model. Later, the fractional grey model has appealed many interesting researches. For example, $\mathrm{Wu}$ et al. [24] proposed a fractional multivariate grey model to forecast electricity consumption of Shandong, China. Ma et al. [25] proposed a novel fractional timedelayed grey model with grey wolf optimizer to predict the natural gas and coal consumption in Chongqing, China. $\mathrm{Wu}$ et al. [26] investigated the novel fractional grey model, namely, $\operatorname{FAGMO}(1,1, k)$, to predict China's nuclear energy consumption. Due to ease of computation of conformable fractional accumulation and difference, Ma et al. [27] introduced it into grey model; as a result, a novel conformable fractional grey model was proposed. Xie et al. [28] proposed a fractional grey model in opposite direction.

Based on the previous literature, this paper proposed a novel grey model which combines a new whitening equation with variable coefficient and the existing $\operatorname{GM}\left(1,1, t^{\alpha}\right)$. The novelties of this paper are drawn as follows: (i) an improved grey model with time term, namely, $\operatorname{OGM}\left(1,1, t^{\alpha}\right)$, is proposed; (ii) the time response function of the model and the restored values are deduced in detail; (iii) the model parameters of the novel model are determined by a well-known algorithm, namely, particle swarm optimization (PSO) [29]; (iv) two examples and a real application are used to verify the effectiveness of the novel model.

The rest of this paper is organized as follows: Section 2 depicts the modelling produces of the existing $\mathrm{GM}\left(1,1, t^{\alpha}\right)$ and the novel model. Section 3 optimizes the parameters of the novel model based on PSO. Section 4 carries out two empirical examples to prove the validity of the novel model. Section 5 applies the novel model to predict oil consumption of China and the main conclusions are listed in the final section.

\section{Methodologies}

2.1. The Traditional $\operatorname{GM}\left(1,1, t^{\alpha}\right)$ Model. The modelling processes of the $\mathrm{GM}\left(1,1, t^{\alpha}\right)$ model proposed by Qian et al. [17] can be described as follows.

Assume a nonnegative series to be

$$
X^{(0)}=\left(x^{(0)}(1), x^{(0)}(2), \ldots, x^{(0)}(n)\right), n \geq 4
$$

and then the first-order accumulative generating operation $(1-\mathrm{AGO})$ series is written as

$$
X^{(1)}=\left(x^{(1)}(1), x^{(1)}(2), \ldots, x^{(1)}(n)\right),
$$

where $x^{(1)}(k)=\sum_{i=1}^{k} x^{(0)}(i)$.

The equation

$$
\frac{d x^{(1)}(t)}{d t}+a x^{(1)}(t)=b t^{\alpha}+c, \alpha>0,
$$

is called the whitening equation of $\operatorname{GM}\left(1,1, t^{\alpha}\right)$. $a$ and $b t^{\alpha}+c$ refer the development coefficient and grey action quantity.

The basic form of $\operatorname{GM}\left(1,1, t^{\alpha}\right)$ is given by

$$
x^{(0)}(k)+a z^{(1)}(k)=b k^{\alpha}+c,
$$

where $z^{(1)}(k)$ is called the background value and $z^{(1)}(k)=0.5 \times\left(x^{(1)}(k)+x^{(1)}(k-1)\right)$.

Using the least square method, the model parameters $a$, $b$, and $c$ can be estimated, there is

$$
(a, b, c)^{T}=\left(B^{T} B\right)^{-1} B^{T} Y
$$

where

$$
\begin{aligned}
& B=\left(\begin{array}{ccc}
-z^{(1)}(2) & 2^{\alpha} & 1 \\
-z^{(1)}(3) & 3^{\alpha} & 1 \\
\vdots & \vdots & \vdots \\
-z^{(1)}(n) & n^{\alpha} & 1
\end{array}\right), \\
& Y=\left(\begin{array}{c}
x^{(0)}(2) \\
x^{(0)}(3) \\
\vdots \\
x^{(0)}(n)
\end{array}\right) .
\end{aligned}
$$

2.2. The Improved $\operatorname{GM}\left(1,1, t^{\alpha}\right)$ Model. Based on the above description of the $\operatorname{GM}\left(1,1, t^{\alpha}\right)$ model, a new whitening equation with a variable coefficient is introduced in (3) so as to improve further the forecasting ability of the $\operatorname{GM}\left(1,1, t^{\alpha}\right)$ model; as a result, the improved $\mathrm{GM}\left(1,1, t^{\alpha}\right)$ model, namely, $\operatorname{OGM}\left(1,1, t^{\alpha}\right)$, is proposed. The modelling procedure of $\operatorname{OGM}\left(1,1, t^{\alpha}\right)$ is summarized as follows.

Definition 1. Let $u>0$; the whitening equation of a new grey forecasting model is given by

$$
\frac{d x^{(1)}(t)}{d t^{u}}+a x^{(1)}(t)=b t^{\alpha}+c, \alpha>0 .
$$

Obviously, (7) turns to be (1) as $u=1$, and so the novel model should be regarded as a general form of $\mathrm{GM}\left(1,1, t^{\alpha}\right)$.

Here, (7) is easily rewritten as

$$
\frac{d x^{(1)}(t)}{d t}+a u t^{u-1} x^{(1)}(t)=b t^{\alpha} u t^{u-1}+c u t^{u-1} .
$$

Integrating both sides of (8) over the interval $[k-1, k]$, one can write 


$$
\begin{aligned}
& \int_{k-1}^{k} d x^{(1)}(t)+\int_{k-1}^{k} a u t^{u-1} x^{(1)}(t) d t \\
& =\int_{k-1}^{k} b t^{\alpha} u t^{u-1} d t+\int_{k-1}^{k} c u t^{u-1} d t
\end{aligned}
$$

By using two-point trapezoidal formula, (9) becomes

$$
x^{(0)}(k)+a z_{1}^{(1)}(k)=b\left(\frac{u}{u+\alpha}\left(k^{u+\alpha}-(k-1)^{u+\alpha}\right)\right)+c\left(k^{u}-(k-1)^{u}\right)
$$

where $z_{1}^{(1)}(k)$ is so-called the background value and $z_{1}^{(1)}(k)=0.5 \times\left(u k^{u-1} x^{(1)}(k)+u(k-1)^{u-1} x^{(1)}(k-1)\right)$.

$$
\begin{aligned}
& G=\left(\begin{array}{ccc}
-z_{1}^{(1)}(2) & \frac{u}{u+\alpha}\left(2^{u+\alpha}-1^{u+\alpha}\right) & 2^{u}-1^{u} \\
-z_{1}^{(1)}(3) & \frac{u}{u+\alpha}\left(3^{u+\alpha}-2^{u+\alpha}\right) & 3^{u}-2^{u} \\
\vdots & \vdots & \vdots \\
-z_{1}^{(1)}(n) \frac{u}{u+\alpha}\left(n^{u+\alpha}-(n-1)^{u+\alpha}\right) & n^{u}-(n-1)^{u}
\end{array}\right) \\
& H=\left(\begin{array}{c}
x^{(0)}(2) \\
x^{(0)}(3) \\
\vdots \\
x^{(0)}(n)
\end{array}\right) .
\end{aligned}
$$

Similar to subsection 2.1, the model parameters $a, b$ and $c$ are calculated through the least square method, there is,

$$
(a, b, c)^{T}=\left(G^{T} G\right)^{-1} G^{T} H .
$$

Theorem 1. Given $X^{(0)}, X^{(1)}$ and $(a, b, c)^{T}$, the solution to of (7) can be given by

$$
x^{(1)}(t)=e^{-a t^{u}}\left(e^{a} x^{(0)}(1)+b \int_{1}^{t} f(u, \tau) d \tau+\frac{c}{a}\left(e^{a t^{u}}-e^{a}\right)\right),
$$

where $f(u, t)=e^{-a t^{u}} u t^{u+\alpha-1}$.

Proof. First of all, multiplying both sides in (7) by $e^{a t^{u}}$, there is,

$$
e^{a t^{u}}\left(\frac{d x^{(1)}(t)}{d t}+a u t^{u-1} x^{(1)}(t)\right)=e^{a t^{u}}\left(b t^{\alpha} u t^{u-1}+c u t^{u-1}\right) .
$$

That is,

$$
\frac{d\left[e^{a t^{u}} x^{(1)}(t)\right]}{d t}=e^{a t^{u}}\left(b t^{\alpha} u t^{u-1}+c u t^{u-1}\right)
$$

Afterward, integrating both sides of (15) over the interval $[1, t]$, then

$$
\int_{1}^{t} d\left[e^{a t^{u}} x^{(1)}(\tau)\right]=\int_{1}^{t} e^{a \tau^{u}}\left(b \tau^{\alpha} u \tau^{u-1}+c u \tau^{u-1}\right) d \tau
$$

By simplifying (16), which yields

$$
e^{a t^{u}} x^{(1)}(t)-e^{a} x^{(0)}(1)=b \int_{1}^{t} f(u, \tau) d \tau+\frac{c}{a}\left(e^{a t^{u}}-e^{a}\right) \text {. }
$$

This completes the proof.

In particular, the integral item $\int_{1}^{t} f(u, \tau) d \tau$ in (13) could be approximately estimated using the following equation:

$$
\int_{1}^{t} f(u, \tau) d \tau \approx \sum_{i=2}^{1000} \triangle_{i} f\left(u, \varepsilon_{i}\right)
$$

where $\triangle_{i}$ is taken as 0.001 for any $i$.

And then set $t=k$, the time response function of the novel model is obtained and the restored values of $X^{(0)}, k=$ $2,3, \ldots$ can be acquired using the first-order inverse accumulative generating operation ( 1 - IAGO), there is, 


$$
\widehat{x}^{(0)}(k)=\widehat{x}^{(1)}(k)-\widehat{x}^{(1)}(k-1) .
$$

\section{Optimization of Parameters by PSO}

To assess the prediction accuracy of the novel model, two statistical indices are employed in this paper, which are the mean absolute percentage error (MAPE) and the root mean square error (RMSE), and defined as follows.

$$
\begin{aligned}
\text { MAPE } & =\frac{1}{n-1}\left|\frac{\widehat{x}^{(0)}(k)-x^{(0)}(k)}{x^{(0)}(k)}\right| \times 100 \%, \\
\text { RMSE } & =\sqrt{\frac{1}{n-1} \sum_{i=2}^{n}\left(\hat{x}^{(0)}(k)-x^{(0)}(k)\right)^{2} .}
\end{aligned}
$$

In the above modelling procedures, the variable coefficient $u$ and time-item coefficient $\alpha$ are assumed to be know. In this regard, this subsection is to determine these parameters, based on a well-known algorithm, namely Particle
Swarm Optimization (PSO) algorithm [29]. this subsection constructs a simply optimization problem for searching the best parameters through minimizing the sum of simulated errors between simulated and actual values, which can be mathematically formulated as follows,

$$
\min _{u, \alpha} \text { MAPE }=\frac{1}{n-1}\left|\frac{\hat{x}^{(0)}(k)-x^{(0)}(k)}{x^{(0)}(k)}\right| \times 100 \% .
$$

The detailed computational steps of the novel model based on PSO are summarized as follows.

Step 1. Initialize population. Set $m=50$ particle and 2-dimensional space, each particle in this search space has initial position $x_{i}=\left(x_{i, 1}, x_{i, 2}\right)$ and velocity $v_{i}=\left(v_{i, 1}, v_{i, 2}\right), i=1,2$, $\ldots, m$.

Step 2. Calculate fitness values using (21).

Step 3. Update velocity and position of each particle using the following equations

$$
\begin{aligned}
& v_{i}=w v_{i}+c_{1} \times \text { rand } \times\left(\text { pbest }-x_{i}\right)+c_{2} \times \text { rand } \times\left(g \text { best }-x_{i}\right), \\
& x_{i}=v_{i}+x_{i},
\end{aligned}
$$

where $w$ refers inertia factor, $c_{1}$ and $c_{2}$ are acceleration factors (generally $c_{1}=c_{2}=2$ ), ran $d$ are random numbers that change in the range of $[0,1]$.

Step 4. Update the best position pbest and the best position of the whole particles gbest.

Step 5. Stopping criteria, if $t \geq T_{\max }$ or the current optimum position meets a pre-set minimum threshold, print the best values, otherwise return to Step 2.

The flowchart of the novel model by PSO are also given in Figure 1 so as to provide a clear visualization.

\section{Validation of the Novel Model}

This section provides two examples to examine the prediction performance of the novel model compared with other commonly-used grey system models, which are the traditional $\mathrm{GM}(1,1)$ model, the discrete grey model $(\operatorname{DGM}(1,1))$, the non-homogeneous grey model $(\operatorname{NHGM}(1,1))$ and the grey model with time-item $\left(\mathrm{GM}\left(1,1, t^{\alpha}\right)\right)$.

Case 1. (Forecasting the output values of the high technology industry of China) This subsection takes the output values of the high technology industry of China [30] as an example to verify the novel model. The raw data set is divided into two groups, the data from 2005 to 2012 are utilized to establish the novel model and competitive models, and the left 2 samples are used to examine the prediction performance.
First of all, the time-item coefficient $\alpha$ of GM $\left(1,1, t^{\alpha}\right)$ and the variable coefficient $u$ and the time-item coefficient $\alpha$ are all determined by PSO, the minimum MAPE values and the corresponding parameters can be seen in Table 1, the tracks of seeking for the optimum values of these parameters are graphed in Figure 2.

It is observed from Table 1 that the RMSE and MAPE values of the novel model are 0.15 and $2.03 \%$ for simulation period, and those of the novel model are 0.22 and $1.32 \%$ for prediction period, respectively. These values are smaller than those of other competitors either in simulation or prediction period, this indicates that the novel model has a relatively higher accuracy in this case.

Case 2. (Forecasting the annual per capita electricity consumption in China) The data, gathered from the literature [12], are taken as the second example to prove the validity of the novel model. Similar to Case 1, the data are empirically grouped into the training and testing sets, the first 13 data are used to build the prediction models and the left 3 observations are used to examine the prediction accuracies of these models. The optimal parameters of the novel model and the $\mathrm{GM}\left(1,1, t^{\alpha}\right)$ model by PSO are listed in Table 2, and the tracks of seeking the best values are plotted in Figure 3.

It is easily seen that the novel model outperforms other competing models due to its lowest RMSE and MAPE values in simulation and prediction period, and so the novel model is suitable for prediction problem of the annual per capita electricity consumption in China at hand. 


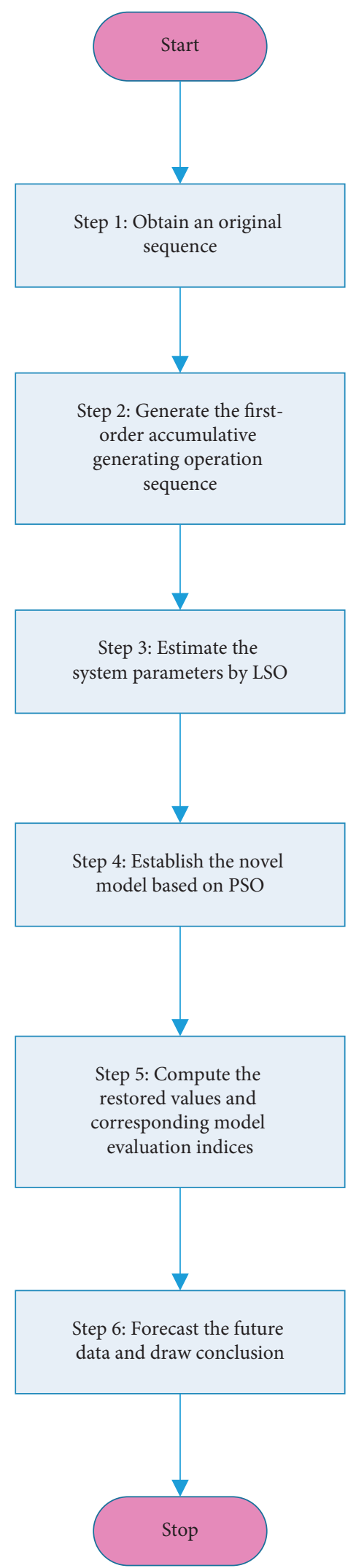

FIgure 1: The flowchart of the novel model by PSO. 
TABLE 1: The simulated results and indices by five grey models.

\begin{tabular}{|c|c|c|c|c|c|c|}
\hline Year & Raw data & $\mathrm{GM}(1,1)$ & $\operatorname{DGM}(1,1)$ & $\operatorname{NHGM}(1,1)$ & $\operatorname{GM}\left(1,1, t^{\alpha}\right)$ & $\operatorname{OGM}\left(1,1, t^{\alpha}\right)$ \\
\hline & & & & & $\alpha=1.37$ & $\begin{array}{c}\alpha=2 \\
u=1.69\end{array}$ \\
\hline 2005 & 3.39 & & & & & \\
\hline 2006 & 4.16 & 4.06 & 4.07 & 4.51 & 4.16 & 4.37 \\
\hline 2007 & 4.97 & 4.72 & 4.73 & 5.11 & 4.73 & 4.94 \\
\hline 2008 & 5.57 & 5.49 & 5.51 & 5.85 & 5.48 & 5.42 \\
\hline 2009 & 5.96 & 6.39 & 6.41 & 6.78 & 6.40 & 6.25 \\
\hline 2010 & 7.45 & 7.44 & 7.46 & 7.94 & 7.48 & 7.42 \\
\hline 2011 & 8.75 & 8.66 & 8.69 & 9.39 & 8.72 & 8.77 \\
\hline 2012 & 10.23 & 10.07 & 10.11 & 11.20 & 10.11 & 10.19 \\
\hline RMSE & & 0.21 & 0.20 & 0.60 & 0.20 & 0.15 \\
\hline MAPE(\%) & & 2.70 & 2.53 & 7.66 & 2.27 & 2.03 \\
\hline 2013 & 11.6 & 11.72 & 11.77 & 13.46 & 11.65 & 11.62 \\
\hline 2014 & 12.74 & 13.64 & 13.69 & 16.28 & 13.34 & 13.05 \\
\hline RMSE & & 0.64 & 0.68 & 2.83 & 0.43 & 0.22 \\
\hline MAPE(\%) & & 4.06 & 4.46 & 21.89 & 2.58 & 1.32 \\
\hline
\end{tabular}

The bold values represent the RMSE and MAPE (\%) of the model proposed in this paper for model evaluation in the example.

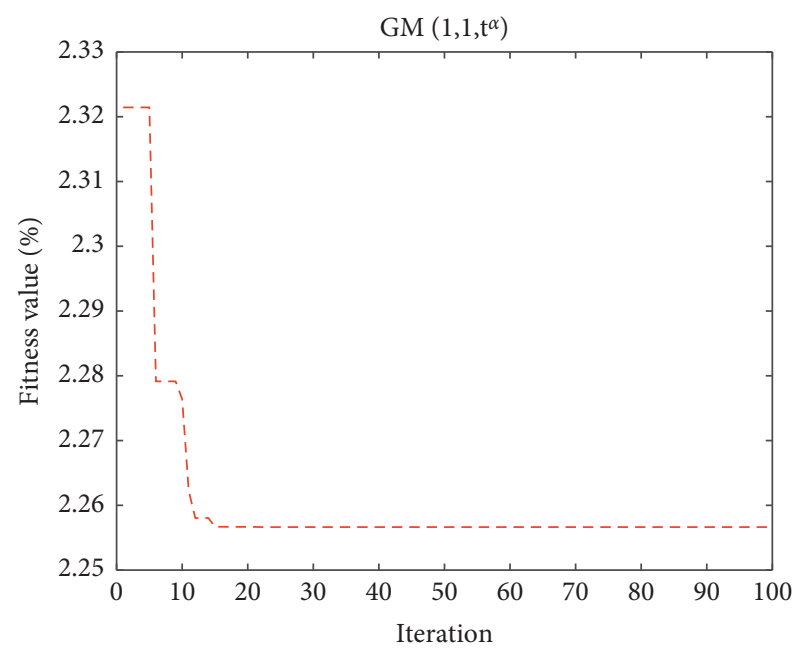

(a)

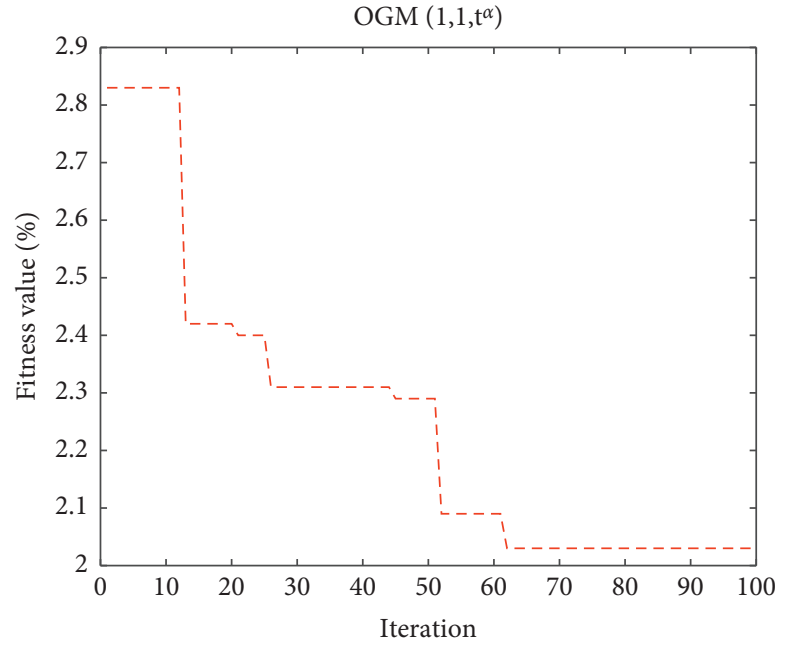

(b)

FIgURE 2: Track of seeking for the optimal parameters of the GM(1,1, $\left.t^{\alpha}\right)$ model (a) and the novel model (b).

\section{Application of Forecasting Oil Consumption of China}

China is currently in a state of developing structural adjustment and steady growth. The manufacturing and service sectors constitute the pillars of the economy. Besides, Economic growth act as the most important factor leading to carbon emission increase, this is because Chinese rapid economic growth in previous decades were heavily dependent on the resource-driven industries, which consumes enormous fossil energy and generates plenty of pollutants. Therefore, accurately forecasting oil consumption becomes crucial in energy systems, especially for fossil-fuel market.

The data of oil consumption of China is collected from the official website National Bureau of Statistics of China (https://data.stats.gov.cn/english), as shown in Table 3. Similar to Case 1 and Case 2 (elaborated in Section 4), the parameters of the novel model and the $\mathrm{GM}\left(1,1, t^{\alpha}\right)$ model are determined by PSO, the minimum MAPE values and the corresponding parameters are given in Table 4, and the tracks of seeking for best values are graphed in Figure 4.

The simulated results can be obtained once the unknown parameters are searched, the prediction results are tabulated in Table 4.

From Table 4, in simulation period, the RMSE values of the novel model and other models are 9.21, 9.21, 10.48, 8.55 and 9.05 , and the MAPE values of these model are $1.75 \%$, $1.75 \%, 2.23 \%, 1.59 \%$ and $1.58 \%$, respectively; in prediction period, the RMSE values of the novel model and other models are $29.87,30.02,12.44,25.73$ and 4.78 , and the MAPE values of these model are $4.46 \%, 4.49 \%, 1.96 \%, 0.83 \%$ and $0.78 \%$, respectively. It is not doubt that the novel model is superior to other competitors, followed by $\operatorname{GM}\left(1,1, t^{\alpha}\right), \operatorname{NHGM}(1,1)$, $\operatorname{GM}(1,1)$ and $\operatorname{DGM}(1,1)$ in this case. The same findings can be found from Figure 5 that fitted values of the novel model are 
TABle 2: The simulated results and indices by five grey models.

\begin{tabular}{|c|c|c|c|c|c|c|}
\hline Year & Raw data & $\operatorname{GM}(1,1)$ & $\operatorname{DGM}(1,1)$ & $\operatorname{NHGM}(1,1)$ & $\operatorname{GM}\left(1,1, t^{\alpha}\right)$ & $\operatorname{OGM}\left(1,1, t^{\alpha}\right)$ \\
\hline & & & & & $\alpha=1.21$ & $\begin{array}{l}\alpha=0.05 \\
u=0.13\end{array}$ \\
\hline 2000 & 1066.9 & & & & & \\
\hline 2001 & 1157.6 & 1265.96 & 1267.79 & 1051.45 & 1133.78 & 1156.14 \\
\hline 2002 & 1286.0 & 1399.87 & 1401.93 & 1236.87 & 1308.18 & 1286.19 \\
\hline 2003 & 1477.1 & 1547.95 & 1550.26 & 1430.35 & 1499.58 & 1476.88 \\
\hline 2004 & 1695.2 & 1711.70 & 1714.28 & 1632.23 & 1704.56 & 1691.54 \\
\hline 2005 & 1913.0 & 1892.77 & 1895.66 & 1842.88 & 1921.28 & 1918.32 \\
\hline 2006 & 2180.6 & 2092.99 & 2096.23 & 2062.68 & 2148.58 & 2153.16 \\
\hline 2007 & 2482.2 & 2314.39 & 2318.02 & 2292.04 & 2385.66 & 2394.35 \\
\hline 2008 & 2607.6 & 2559.21 & 2563.28 & 2531.36 & 2631.94 & 2641.11 \\
\hline 2009 & 2781.7 & 2829.92 & 2834.49 & 2781.07 & 2886.98 & 2893.01 \\
\hline 2010 & 3134.8 & 3129.28 & 3134.39 & 3041.64 & 3150.46 & 3149.83 \\
\hline 2011 & 3497.0 & 3460.30 & 3466.02 & 3313.53 & 3422.11 & 3411.43 \\
\hline 2012 & 3684.2 & 3826.34 & 3832.75 & 3597.23 & 3701.75 & 3677.73 \\
\hline RMSE & & 87.80 & 88.24 & 104.30 & 49.92 & 49.68 \\
\hline MAPE(\%) & & 3.71 & 3.71 & 4.18 & 1.58 & 1.15 \\
\hline 2013 & 3993.0 & 4231.09 & 4238.27 & 3893.26 & 3989.20 & 3948.69 \\
\hline 2014 & 4132.9 & 4678.67 & 4686.70 & 4202.15 & 4284.35 & 4224.26 \\
\hline 2015 & 4321.0 & 5173.58 & 5182.57 & 4524.46 & 4587.10 & 4504.43 \\
\hline RMSE & & 600.42 & 608.04 & 136.80 & 176.78 & 121.04 \\
\hline MAPE(\%) & & 12.97 & 13.16 & 2.96 & 3.31 & 2.52 \\
\hline
\end{tabular}

$\operatorname{GM}\left(1,1, t^{\alpha}\right)$

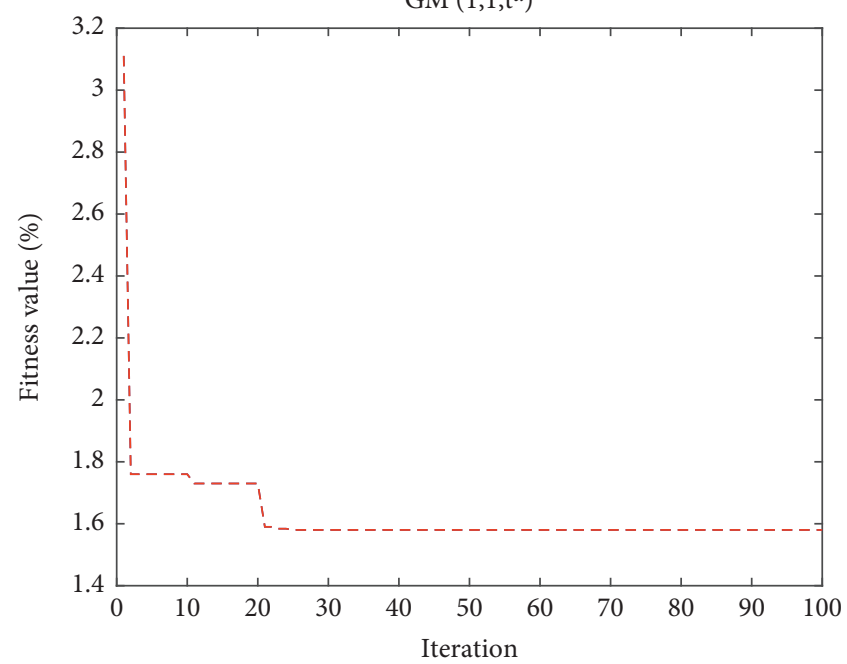

(a)

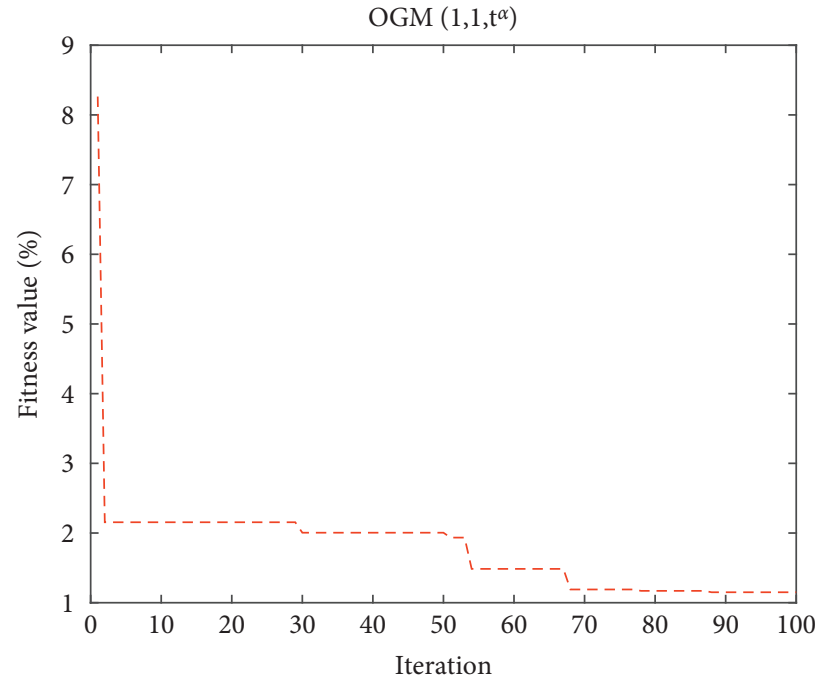

(b)

FIgURE 3: Track of seeking for the optimal parameters of the $\mathrm{GM}\left(1,1, t^{\alpha}\right)$ model (a) and the novel model (b).

TABle 3: Annual oil consumption of China form 2004 to 2018 (Mote).

\begin{tabular}{lccc}
\hline Year & Raw data & Year & Raw data \\
\hline 2004 & 322.6 & 2012 & 487.6 \\
2005 & 328.1 & 2013 & 508.9 \\
2006 & 352.1 & 2014 & 529.5 \\
2007 & 369.6 & 2015 & 561.8 \\
2008 & 376.8 & 2016 & 574.0 \\
2009 & 393.6 & 2017 & 597.5 \\
2010 & 448.9 & 2018 & 628.0 \\
2011 & 465.6 & & \\
\hline
\end{tabular}


TABLE 4: The simulated results and indices by five grey models.

\begin{tabular}{|c|c|c|c|c|c|c|}
\hline Year & Raw data & $\mathrm{GM}(1,1)$ & $\operatorname{DGM}(1,1)$ & $\operatorname{NHGM}(1,1)$ & $\operatorname{GM}\left(1,1, t^{\alpha}\right)$ & $\operatorname{OGM}\left(1,1, t^{\alpha}\right)$ \\
\hline & & & & & $\alpha=1.25$ & $\alpha=1.25$ \\
\hline & & & & & & $u=0.20$ \\
\hline 2004 & 322.6 & & & & & \\
\hline 2005 & 328.1 & 329.39 & 329.53 & 321.90 & 328.47 & 328.40 \\
\hline 2006 & 352.1 & 347.82 & 347.96 & 341.92 & 345.25 & 345.35 \\
\hline 2007 & 369.6 & 367.28 & 367.42 & 362.62 & 365.67 & 367.40 \\
\hline 2008 & 376.8 & 387.82 & 387.97 & 384.04 & 388.16 & 390.72 \\
\hline 2009 & 393.6 & 409.52 & 409.66 & 406.20 & 411.81 & 414.38 \\
\hline 2010 & 448.9 & 432.43 & 432.58 & 429.12 & 436.04 & 438.11 \\
\hline 2011 & 465.6 & 456.62 & 456.77 & 452.83 & 460.47 & 461.79 \\
\hline 2012 & 487.6 & 482.17 & 482.31 & 477.36 & 484.83 & 485.39 \\
\hline 2013 & 508.9 & 509.14 & 509.29 & 502.74 & 508.91 & 508.92 \\
\hline 2014 & 529.5 & 537.62 & 537.77 & 528.99 & 532.59 & 532.38 \\
\hline RMSE & & 9.21 & 9.21 & 10.48 & 8.55 & 9.05 \\
\hline MAPE(\%) & & 1.75 & 1.75 & 2.23 & 1.59 & 1.58 \\
\hline 2015 & 561.8 & 567.70 & 567.84 & 556.15 & 555.76 & 555.79 \\
\hline 2016 & 574.0 & 599.45 & 599.60 & 584.24 & 578.36 & 579.16 \\
\hline 2017 & 597.5 & 632.99 & 633.13 & 613.30 & 600.35 & 602.49 \\
\hline 2018 & 628.0 & 668.40 & 668.54 & 643.36 & 621.69 & 625.82 \\
\hline RMSE & & 29.87 & 30.02 & 12.44 & 25.73 & 4.78 \\
\hline MAPE(\%) & & 4.46 & 4.49 & 1.96 & 0.83 & 0.78 \\
\hline
\end{tabular}

The bold values represent the RMSE and MAPE (\%) of the model proposed in this paper for model evaluation in the example.

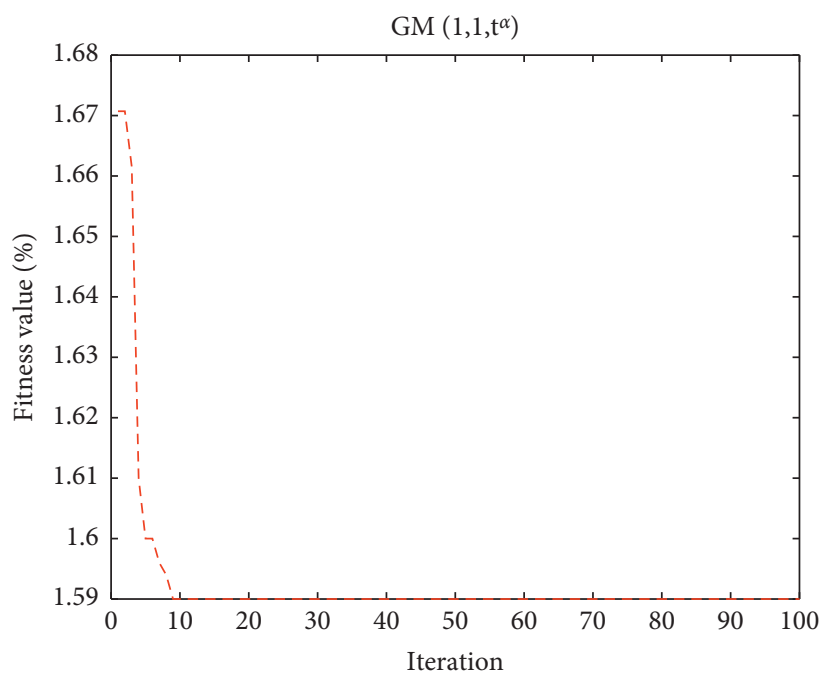

(a)

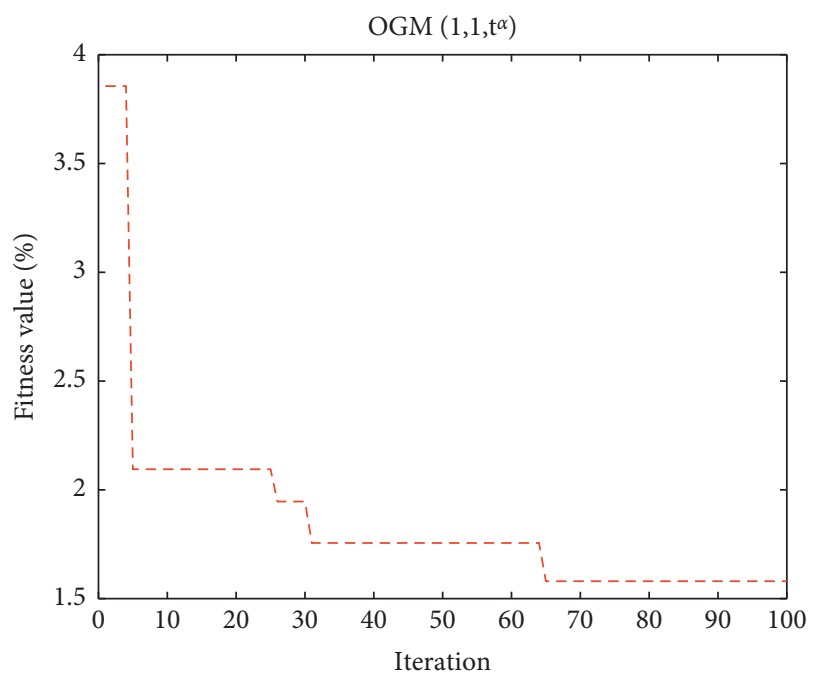

(b)

FIgURE 4: Track of seeking for the optimal parameters of the GM(1,1, $\left.t^{\alpha}\right)$ model (a) and the novel model (b).

much closer than those of other models. In summary, the proposed model is appreciated model for forecasting oil consumption of China so as to provide a valuable reference for energy sectors and related enterprises, which asstanting decision-makers understand future information and formulate corresponding strategies in advance. 


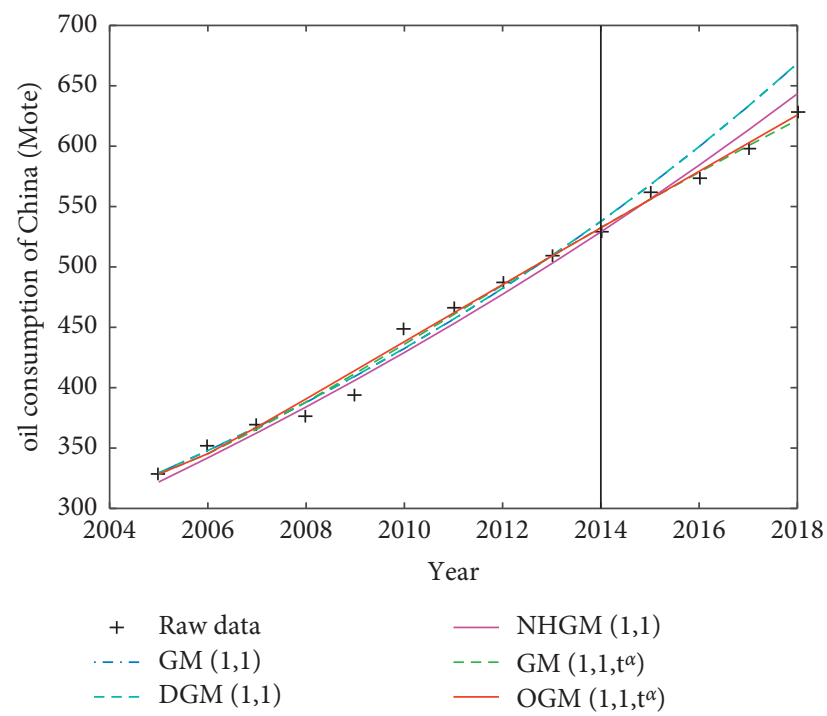

Figure 5: Curves of raw data and simulated values in oil consumption of China from 2004 to 2018.

\section{Conclusion and Future Work}

Aimed to improve further the existing grey model with time item, abbreviated as $\operatorname{GM}\left(1,1, t^{\alpha}\right)$, a new whitening equation with variable coefficient is introduced for acquiring extensive whitening equation, as a consequence, an improved grey model with time item, namely $\operatorname{OGM}\left(1,1, t^{\alpha}\right)$, is proposed. The model has many advantages. First, $\operatorname{OGM}\left(1,1, t^{\alpha}\right)$ can be directly extended to fractional order. In addition, the background value coefficient can be adjusted dynamically. Accordingly, it has a good scalability and can be directly extended to other new types of models. Second, OGM $\left(1,1, t^{\alpha}\right)$ has a simple structure and relatively few parameters, and the time consumption is usually tiny. For example, OGM $\left(1,1, t^{\alpha}\right)$ is implemented in MATLAB environment on a computer with windows 10 system, Intel i5 $\mathrm{CPU}$, and $8 \mathrm{~GB}$ of RAM. The time costs of the first case and the second case are only $0.029934 \mathrm{~s}$ and $0.047817 \mathrm{~s}$, respectively. Thus, the model does not have the problem of occupying large computing resources.

The main contributions of this paper can be summarized as follows.

(1) An improved $\mathrm{GM}\left(1,1, t^{\alpha}\right)$ is put forward, which is based on a novel whitening equation with variable coefficient.

(2) PSO algorithm is utilized to determine the unknown parameters, which are variable coefficient of the whitening equation and time-item parameter.

(3) $\operatorname{GM}\left(1,1, t^{\alpha}\right)$ has a good scalability and can be directly extended to other new types of models.

(4) The novel model is applied to predict the oil consumption of China due to its flexible forecasting ability.

The scalability of this model is very good. For example, the grey model based on the proposed method can be directly extended to fractional order. In addition, the background value coefficient can be adjusted dynamically.
Although the superiorities of the novel model have been discussed, there exist some issue, which should be noticed. For example, the inherent error caused by leap from the whitening equation to the basic form should be considered in the coming research. Besides, the fractional accumulation would be received more attention in next work.

\section{Abbreviations \\ AGO: Accumulative generating operation \\ PSO: Particle swarm optimization \\ MAP: $\quad$ Mean absolute percentage error \\ RMSE: Root mean square error \\ GM: $\quad$ Grey model \\ DGM: Discrete grey model \\ NHGM: Non-homogeneous grey model}

\section{Symbols}

$z^{1}(k): \quad$ Background value

$X^{(0)}: \quad \quad$ A non-negative series

$x^{(1)}: \quad$ First-order accumulative generating

a: $\quad$ Development coefficient

$b t^{x A} 6 x C 1+c$ : Grey action quantity

$x_{i}: \quad$ Particle position

$v_{i}: \quad$ Searching velocity

$w: \quad$ Refers inertia factor

$c_{1}: \quad$ Acceleration factor

$c_{2}: \quad$ Acceleration factor

rand: Random number.

\section{Data Availability}

The data used to support the findings of this study are included within the article. 


\section{Conflicts of Interest}

The authors declare that there are no conflicts of interest regarding the publication of this paper.

\section{Acknowledgments}

This work was supported by the National Natural Science Foundation of China (Grant no. 11661 001) and Natural Science Foundation of Guangxi (Grant no. 2016GXNSFBA380069).

\section{References}

[1] L. Pei, Q. Li, and Z. Wang, “The NLS-based nonlinear grey Bernoulli model with an application to employee demand prediction of high-tech enterprises in China," Grey Systems: Theory and Application, vol. 8, no. 2, pp. 133-143, 2018.

[2] J. L. Deng, "Control problems of grey systems," Systems \& Control Letters, vol. 1, no. 5, pp. 288-294, 1982.

[3] Y. Li, J. Wang, D. Zhao, G. Li, and C. Chen, "A two-stage approach for combined heat and power economic emission dispatch: combining multi-objective optimization with integrated decision making," Energy, vol. 162, pp. 237-254, 2018.

[4] S. Ding, "A novel self-adapting intelligent grey model for forecasting China's natural-gas demand," Energy, vol. 162, pp. 393-407, 2018.

[5] Z.-X. Wang and Q. Li, "Modelling the nonlinear relationship between $\mathrm{CO} 2$ emissions and economic growth using a PSO algorithm-based grey Verhulst model," Journal of Cleaner Production, vol. 207, pp. 214-224, 2019.

[6] L. Wu, S. Liu, D. Liu, Z. Fang, and H. Xu, "Modelling and forecasting CO2 emissions in the BRICS (Brazil, Russia, India, China, and South Africa) countries using a novel multivariable grey model," Energy, vol. 79, pp. 489-495, 2015.

[7] U. Kumar and V. K. Jain, "Time series models (Grey-Markov, Grey Model with rolling mechanism and singular spectrum analysis) to forecast energy consumption in India," Energy, vol. 35, no. 4, pp. 1709-1716, 2010.

[8] G. M. Duman, E. Kongar, and S. M. Gupta, "Estimation of electronic waste using optimized multivariate grey models," Waste Management, vol. 95, pp. 241-249, 2019.

[9] X. Ma and Z. Liu, "Application of a novel time-delayed polynomial grey model to predict the natural gas consumption in China," Journal of Computational and Applied Mathematics, vol. 324, pp. 17-24, 2017.

[10] N.-M. Xie and S.-F. Liu, "Discrete grey forecasting model and its optimization," Applied Mathematical Modelling, vol. 33, no. 2, pp. 1173-1186, 2009.

[11] L. Dang and W. Baolei, "Grey forecasting model with polynomial term and its optimization," Optimization, vol. 29, no. 3, pp. 58-69, 2017.

[12] B. Wei, N. Xie, and A. Hu, "Optimal solution for novel grey polynomial prediction model," Applied Mathematical Modelling, vol. 62, pp. 717-727, 2018.

[13] Z.-X. Wang, Q. Li, and L.-L. Pei, "A seasonal GM(1,1) model for forecasting the electricity consumption of the primary economic sectors," Energy, vol. 154, pp. 522-534, 2018.

[14] Z.-X. Wang, Q. Li, and L.-L. Pei, "Grey forecasting method of quarterly hydropower production in China based on a data grouping approach," Applied Mathematical Modelling, vol. 51, pp. 302-316, 2017.
[15] W.-Z. Wu and T. Zhang, "An improved gray interval forecast method and its application," Communications in Statistics Theory and Methods, vol. 49, no. 5, pp. 1120-1131, 2020.

[16] Y.-Y. Chen, H.-T. Liu, and H.-L. Hsieh, "Time series interval forecast using $\operatorname{GM}(1,1)$ and $\operatorname{NGBM}(1,1)$ models," Soft Computing, vol. 23, no. 5, pp. 1541-1555, 2019.

[17] W. Qian, Y. Dang, and S. Liu, "Grey GM $\left(1,1, t^{\alpha}\right)$ model with time power and its application," Systems Engineering \& Practice, vol. 32, pp. 2247-2252, 2012.

[18] C.-I. Chen, H. L. Chen, and S.-P. Chen, "Forecasting of foreign exchange rates of Taiwan's major trading partners by novel nonlinear Grey Bernoulli model NGBM(1,1)," Communications in Nonlinear Science and Numerical Simulation, vol. 13, no. 6, pp. 1194-1204, 2008.

[19] Z.-X. Wang, K. W. Hipel, Q. Wang, and S.-W. He, "An optimized $\operatorname{NGBM}(1,1)$ model for forecasting the qualified discharge rate of industrial wastewater in China," Applied Mathematical Modelling, vol. 35, no. 12, pp. 5524-5532, 2011.

[20] W. Z. Wu, T. Zhang, and C. L. Zheng, "A novel optimized nonlinear grey Bernoulli model for forecasting China's GDP," Complexity, vol. 2019, Article ID 1731262, 10 pages, 2019.

[21] N. T. Nguyen, V. T. Phan, and Z. Malarad, "Nonlinear grey Bernoulli model based on fourier transformation and its application in forecasting the electricity consumption in vietnam," Journal of Intelligent and Fuzzy Systems, vol. 37, pp. 1-11, 2019, Preprint.

[22] X. Ma, Z. Liu, and Y. Wang, "Application of a novel nonlinear multivariate grey Bernoulli model to predict the tourist income of China," Journal of Computational and Applied Mathematics, vol. 347, pp. 84-94, 2019.

[23] L. Wu, S. Liu, L. Yao, S. Yan, and D. Liu, "Grey system model with the fractional order accumulation," Communications in Nonlinear Science and Numerical Simulation, vol. 18, no. 7, pp. 1775-1785, 2013.

[24] L. Wu, X. Gao, Y. Xiao, Y. Yang, and X. Chen, "Using a novel multi-variable grey model to forecast the electricity consumption of Shandong Province in China," Energy, vol. 157, pp. 327-335, 2018.

[25] X. Ma, X. Mei, W. Wu, X. Wu, and B. Zeng, "A novel fractional time delayed grey model with Grey Wolf Optimizer and its applications in forecasting the natural gas and coal consumption in Chongqing China," Energy, vol. 178, pp. 487-507, 2019.

[26] W. Wu, X. Ma, B. Zeng, Y. Wang, and W. Cai, "Application of the novel fractional grey model FAGMO $(1,1, \mathrm{k})$ to predict China's nuclear energy consumption," Energy, vol. 165, pp. 223-234, 2018.

[27] X. Ma, W. Wu, B. Zeng, Y. Wang, and X. Wu, "The conformable fractional grey system model," ISA Transactions, vol. 96, pp. 255-271, 2020.

[28] W. Xie, C. Liu, and W. Z. Wu, "The fractional non-equidistant grey opposite-direction model with time-varying characteristics," Soft Computing, vol. 24, pp. 1-10, 2020.

[29] J. Kennedy and R. Eberhart, "Particle swarm optimization," in Proceedings of the International Conference on Neural Networks, pp. 1942-1948, Perth, WA, Australia, November, 1995.

[30] S. Ding, Y. G. Dang, X. U. Ning, L. Wei, and Y. E. Jing, "Multivariable time-delayed discrete grey model," Control and Decision, vol. 32, pp. 199-202, 2017. 